\title{
Analysis of the Knockdown Resistance Locus (kdr) in Anopheles stephensi, An. arabiensis, and Culex pipiens s.l. for Insight Into the Evolution of Target-site Pyrethroid Resistance in Eastern Ethiopia
}

\author{
Tamar E. Carter, ${ }^{1 *}$ Araya Gebresilassie, ${ }^{2}$ Shantoy Hansel, ${ }^{3}$ Lambodhar Damodaran, ${ }^{4}$ Callum Montgomery, ${ }^{3}$ Victoria Bonnell, ${ }^{5}$ \\ Karen Lopez, ${ }^{3}$ Daniel Janies, ${ }^{3 \dagger}$ and Solomon Yared ${ }^{6 \dagger}$ \\ ${ }^{1}$ Department of Biology, Baylor University, Waco, Texas; '2Department of Zoological Sciences, Addis Ababa University, Addis Ababa, Ethiopia; \\ ${ }^{3}$ Department of Bioinformatics and Genomics, University of North Carolina at Charlotte, Charlotte, North Carolina; ${ }^{4}$ Institute of Bioinformatics, \\ University of Georgia, Athens, Georgia; ${ }^{5}$ Department of Molecular Biology and Biochemistry, Pennsylvania State University, State College, \\ Pennsylvania; ${ }^{6}$ Department of Biology, Jigjiga University, Jigjiga, Ethiopia
}

\begin{abstract}
The malaria vector, Anopheles stephensi, which is typically restricted to South Asia and the Middle East, was recently detected in the Horn of Africa. Addressing the spread of this vector could involve integrated vector control that considers the status of insecticide resistance of multiple vector species in the region. Previous reports indicate that the knockdown resistance mutations $(k d r)$ in the voltage-gated sodium channel $(v g s c)$ are absent in both pyrethroidresistant and pyrethroid-sensitive An. stephensi in eastern Ethiopia; however, similar information about other vector species in the same areas is limited. In this study, $k d r$ and the neighboring intron were analyzed in An. stephensi, An. arabiensis, and Culex pipiens s.l. collected between 2016 and 2017 to determine the evolutionary history of $k d r$ in eastern Ethiopia. A sequence analysis revealed that all of Cx. pipiens s.I. $(N=42)$ and $71.6 \%$ of the An. arabiensis $(N=67)$ carried $k d r$ L1014F, which is known to confer target-site pyrethroid resistance. Intronic variation was only observed in An. stephensi (six segregating sites, three haplotypes), which was previously shown to have no kdr mutations. In addition, no evidence of non-neutral evolutionary processes was detected at the An. stephensi kdr intron, thereby further supporting the target-site mechanism not being a major resistance mechanism in this An. stephensi population. Overall, these results show key differences in the evolution of target-site pyrethroid/dichlorodiphenyltrichloroethane resistance mutations in populations of vector species from the same region. Variations in insecticide resistance mechanism profiles between eastern Ethiopian mosquito vectors may lead to different responses to insecticides used in integrated vector control.
\end{abstract}

\section{INTRODUCTION}

Vector-borne diseases are a major public health concern; of these, malaria remains a leading threat, with 229 million cases reported in 2019. ${ }^{1}$ In Ethiopia, where both Plasmodium vivax and $P$. falciparum are prevalent and multiple Anopheles vector populations are present, 2.6 million malaria cases were reported in 2019. ${ }^{1}$ Malaria control in Ethiopia and the rest of Africa is now challenged with the recent discovery of An. stephensi, a malaria vector that is typically restricted to South Asia and the Middle East, in the Horn of Africa that recently demonstrated local transmission of Plasmodium. ${ }^{2-5}$ Among several approaches to mitigating the spread of An. stephensi is integrated vector control that targets multiple vectors. Integrated vector control has the benefits of reducing costs and minimizing adverse outcomes of single-target vector control for nontarget species populations. ${ }^{6}$

Integrated vector control strategies based on insecticides should account for insecticide resistance status of the different vectors. In Ethiopia, insecticides like pyrethroids have been deployed through indoor residual spraying and longlasting insecticidal nets (LLIN). Exacerbated by the use of insecticides in agricultural industries, widespread insecticide resistance has evolved and has been reported across multiple vector species. ${ }^{7}$ In Culicidae, the main mechanisms of resistance to pyrethroids include target-site and metabolicbased resistance. ${ }^{8}$ Pyrethroid-based target-site resistance is

\footnotetext{
${ }^{*}$ Address correspondence to Tamar E. Carter, Department of Biology, Baylor University, Waco, TX 76798-7344. E-mail: tamar_ carter@baylor.edu
}

†These authors contributed equally to this work. caused by mutations in the voltage-gated sodium channel that lead to an altered neurological response to insecticides in mosquitoes (i.e., knockdown resistance $[k d r]){ }^{9}$ Knockdown resistance is broadly studied and is widely reported across species of Culicidae, including Anopheles spp. ${ }^{8}$ and Culex pipiens s.l. ${ }^{10}$ Regarding Anopheles, kdr involves the substitution of leucine (TTA) with phenylalanine (TTT) or serine (TCA) in the voltage-gated sodium channel protein, commonly known as $k d r$ mutations L1014F and L1014S. ${ }^{11}$ Similar mutations that confer resistance to pyrethroids (also known as L1014F and L1014S) are observed in the vgsc of Culex mosquitoes.

To achieve metabolic resistance, the insecticide is degraded, sequestered, or exported out of the cell before it can bind to its target. ${ }^{8}$ Metabolic resistance has not been linked to a single trackable genetic variant in most species. However, previous functional studies have found that overexpression of detoxification enzymes such as cytochrome $\mathrm{P} 450$ s leads to metabolic resistance. ${ }^{8,12}$

In Ethiopia, pyrethroid resistance and dichlorodiphenyltrichloroethane (DDT) resistance have been reported for the primary malaria vector An. arabiensis in much of the northern and western portions of the country. ${ }^{13-16}$ Regarding An. arabiensis, both target-site and metabolic resistance have a role in pyrethroid resistance and DDT resistance. In eastern Ethiopia, a recent investigation revealed that An. stephensi was resistant to pyrethroids and DDT (consistent with findings outside of Ethiopia ${ }^{17}$ ); however, the L1014F and L1014S mutations were absent. ${ }^{18} \mathrm{An}$. arabiensis insecticide resistance in eastern Ethiopia has not been well-characterized. Furthermore, the status of insecticide resistance in $C x$. pipiens s.l. (most likely $C x . p$. quinquefasciatus) is unknown throughout most of the country. 
Knowing the status of resistance to pyrethroids across vector species in a region can provide insight into the effectiveness of particular insecticides used to target multiple species. Genetic analyses of putative insecticide resistance loci across local vector populations can provide information about the range of mechanisms of insecticide resistance in a region. Although $k d r$ L1014F and L1014S mutation frequencies provide preliminary evidence of target-site resistance to pyrethroids, an analysis of the variations in neighboring intronic regions provides information about the long-term impact of pyrethroids on the evolution of the mosquito populations. Tests of neutrality ${ }^{19}$ can be used to evaluate the genetic diversity of the $k d r$ locus, including the intronic region, to determine if the patterns differ from expectations under neutral evolution. It is expected that if the $k d r$ locus undergoes selection because of pressure from the pyrethroids, then we would hypothesize that a selective sweep would lead to decreased nucleotide diversity of linked alleles via hitchhiking. ${ }^{20,21}$ Therefore, these analyses are helpful to clarifying the mechanisms of resistance, clarifying the current status of pyrethroid resistance, and predicting the risk of resistance emerging locally. We examined the nucleotide diversity surrounding the $k d r$ locus to test the hypothesis of selective sweeps in An. stephensi, An. arabiensis, and Culex pipiens s.l. collected in eastern Ethiopia.

\section{METHODS}

This study involved sequencing of a portion of the vgsc gene that contains loci that, when mutated, confer resistance to pyrethroids. For An. stephensi, data were from sequences generated during a previous study ${ }^{18}$ and the present study. An. arabiensis and Culex sequence data were also generated during this study.

Sample collection and species identification. An. stephensi was collected from Kebri Dehar during the 2016 survey that resulted in the first identification of the species in Ethiopia. ${ }^{3}$ Mosquitoes were collected as larvae and laboratory-reared for testing for resistance to insecticides as previously detailed. ${ }^{18} \mathrm{An}$. arabiensis and Culex specimens collected in eastern Ethiopia in 2017 were included in this study. An. arabiensis species identification was based on morphological keys and molecular analysis of internal transcribed spacer 2 (ITS2) and cytochrome oxidase I (COI) loci, as reported previously. ${ }^{22}$ An. arabiensis were collected using CDC light traps (John W. Hock, Gainesville, FL) over four different collection times at two sites, Meki (east-central Ethiopia) and Harewe (east) in 2017. Harewe and Meki are approximately $350 \mathrm{~km}$ and $600 \mathrm{~km}$ northwest of Kebri Dehar, respectively (Figure 1).

Culex specimens were collected using CDC light traps in Kebri Dehar in 2017. The morphological key and sequencing of the ITS2 locus were used for Culex identification using a previously published polymerase chain reaction (PCR) protocol. $^{3}$ All amplicons were cleaned using Exosap and sequenced using Sanger technology with ABI BigDyeTM Terminator version 3.1 chemistry (Thermo Fisher, Santa Clara, CA) according to recommendations of the manufacturer and run on a 3130 Genetic Analyzer (Thermo Fisher). Sequences were cleaned and analyzed using CodonCode Aligner Program version 6.0.2 (CodonCode Corporation, Centerville, MA). ITS2 sequences from Culex specimen were submitted as queries to the National Center for Biotechnology Information's Basic Local Alignment Search Tool for species identification. ${ }^{23}$

Amplification and sequencing of kdr loci. When species identification or species complex identification was complete, samples were processed. For the kdr mutation analysis, PCR was used to amplify the region of the vgsc gene that housed the homologous $k d r 1014$ and a neighboring downstream intron in all specimens (reference sequences used for An. stephensi, An. arabiensis, and Culex pipiens s.l. were JF304952, GU248311, and BN001092, respectively). One leg from each mosquito specimen or extracted DNA

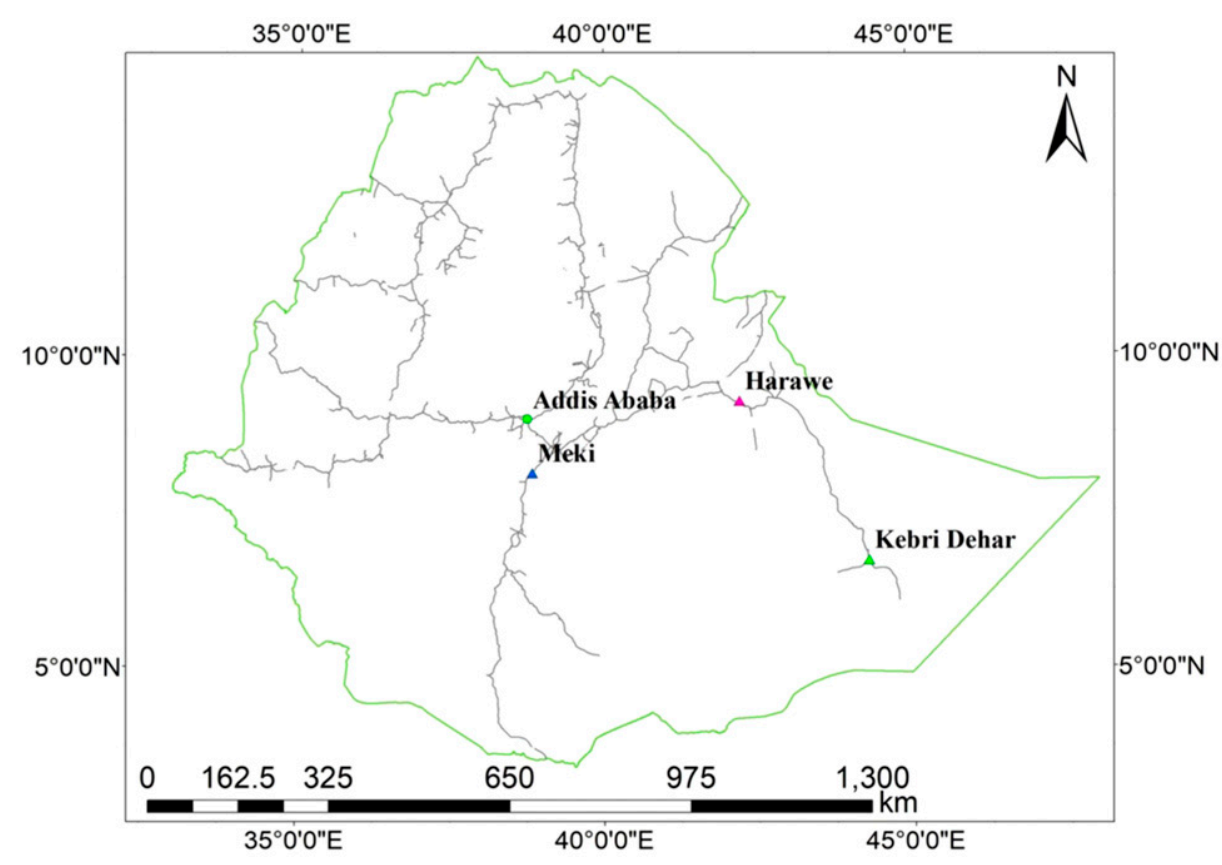

FIGURE 1. Collection sites. This figure appears in color at www.ajtmh.org. 
was used as an individual template for PCR. Each species required a different $\mathrm{PCR}$ protocol. DNA extraction were performed using DNeasy Qiagen kit (Qiagen, Valencia, CA). All PCRs were performed with $25 \mu \mathrm{L}$ with $12.5 \mu \mathrm{L} 2 \mathrm{X}$ Promega Hot Start Master Mix (Promega Corporation, Madison, WI) and the primer conditions listed in Table 1. An. stephensi kdr amplification was completed according to Singh et al., ${ }^{24}$ with modifications detailed by Yared et al. ${ }^{18}$ Temperature cycling was as follows: $95^{\circ} \mathrm{C}$ for 5 minutes, followed by 35 cycles of $95^{\circ} \mathrm{C}$ for 30 seconds, $50^{\circ} \mathrm{C}$ for 30 seconds, $72^{\circ} \mathrm{C}$ for 45 seconds, and a final extension of $72^{\circ} \mathrm{C}$ for 7 minutes. Amplifications of the $k d r$ fragment from An. arabiensis were completed according to the methods of Verhaeghen et al. ${ }^{25}$ Temperature cycling was as follows: $95^{\circ} \mathrm{C}$ for 1 minute, followed by 30 cycles of $95^{\circ} \mathrm{C}$ for 30 seconds, $52^{\circ} \mathrm{C}$ for $30 \mathrm{sec}-$ onds, $72^{\circ} \mathrm{C}$ for 1 minute, and a final extension of $72^{\circ} \mathrm{C}$ for 10 minutes. Amplifications of the $k d r$ fragment from Culex pipiens s.l. were completed according to the methods of Chen et al. ${ }^{26}$ Temperature cycling was as follows: $94^{\circ} \mathrm{C}$ for 5 minutes, followed by 30 cycles of $94^{\circ} \mathrm{C}$ for 40 seconds, $58^{\circ} \mathrm{C}$ for 30 seconds, $72^{\circ} \mathrm{C}$ for 40 seconds, and a final extension of $72^{\circ} \mathrm{C}$ for 8 minutes.

All amplicons were cleaned using Exosap and sequenced using Sanger technology with ABI BigDyeTM Terminator version 3.1 chemistry (Thermo Fisher) according to the manufacturer's recommendations and run on a 3130 Genetic Analyzer (Thermo Fisher).

Sequence analysis. Sequences were submitted as queries to the National Center for Biotechnology Information's Basic Local Alignment Search Tool to confirm that the correct loci were amplified. Sequences were then aligned in CodonCode (CodonCode Corp., Dedham, MA) according to species or species complex to identify kdr L1014F or L1014S mutations based on reference sequence details from previous reports. ${ }^{18,24,25}$ Heterozygous genotypes at $k d r$ were determined based on the number of peaks observed in the chromatogram, with each peak indicating different alleles. The $k d r$ allele and genotype frequencies were then calculated and compared across species.

We determined the level of diversity in the neighboring intron downstream of the kdr 1014 in Culex spp., An. arabiensis, and An. stephensi for additional evidence of selection on that locus. In addition to the sequences generated in this study, we included sequences from resistant and nonresistant An. stephensi analyzed during a previous study of insecticide resistance in An. stephensi. ${ }^{18}$ We calculated the number of segregating sites, nucleotide diversity, estimated number of haplotypes, and haplotype diversity using the program DNAsp version $5 .^{27}$ Haplotypes were reconstructed using Phase 2.1, ${ }^{28}$ HAPAR, and fastPHASE ${ }^{29}$ algorithms in DNAsp. The neighboring downstream intron was also tested for neutrality using Tajima's D, ${ }^{19}$ Fu's $F,{ }^{30}$ and Fu and Li's D* and $\mathrm{F}^{\star}$ tests. $^{31}$

\section{RESULTS}

Before insecticide resistance genotyping, all Culex ITS2 sequences were analyzed to identify species. All sequences were identical and had equivalent high matching scores for two members of the $C x$. pipiens complex: $C x$. p. quinquefasciatus and $C x . p$. pipiens. Because we could not identify the species of these mosquitos, we refer to these specimens by the broader taxonomic classification, Cx. pipiens s.l. (i.e., Cx. pipiens complex), in this study. An. arabiensis species identification was detailed in a previous study. ${ }^{22}$ Totals of 10, 33, and 24 An. arabiensis were collected in Harewe in November 2016, Harewe in July/August 2017, and Meki in July 2017, respectively.

The $k d r$ analysis. The $k d r$ fragments were sequenced for An. stephensi, Cx. pipiens s.l., and An. arabiensis. The sequencing resulted in 184, 452, and 290 basepair fragments for An. stephensi, Cx. pipiens s.I., and An. arabiensis, respectively. The percent of each $k d r$ genotype observed by species is shown in Figure 2. A total of 131 An. stephensi were analyzed, including 80 newly reported sequences. None of the An. stephensi analyzed during this study had a mutation at $k d r$ 1014. All 42 Cx. pipiens s.l. specimens collected at the same site carried $k d r$ L1014F mutations as homozygous. Of the $67 \mathrm{An}$. arabiensis, $71.6 \%$ carried the $k d r$ L1014F mutation (heterozygous and homozygous). The allele frequency of L1014F mutation varied across An. arabiensis collections, in which the highest frequency was observed in Harewe in November 2016 (100\%). The L1014F allele frequencies for the Harewe July/August 2017 and Meki July 2017 collections were $86.4 \%$ and $10 \%$, respectively. No L1014S mutations were detected in Cx. pipiens s.l. or An. arabiensis.

Some of the neighboring downstream introns for each species were analyzed to evaluate the level of diversity (Figure 3). The intron analysis revealed no polymorphisms for either Cx. pipiens or An. arabiensis (for both L1014F and L1014 wild type specimens). Of the $131 \mathrm{An}$. stephensi specimens from Kebri Dehar examined for $k d r$ mutations, six segregating sites were detected and three haplotypes were predicted. Genetic diversity estimates are reported in Table 2.

To further evaluate the potential functional significance of the $k d r$ locus in An. stephensi based on evidence of positive selection, we performed neutrality tests at the An. stephensi $k d r$ intron. No evidence of non-neutral processes was detected in An. stephensi for the $k d r$ locus (Table 3). The absence of variations in An. arabiensis and Cx. pipiens s.l. $k d r$ introns precluded neutrality tests.

TABLE 1

List of primers and conditions used for polymerase chain reaction amplification of portions of the voltage-gated sodium channel gene

\begin{tabular}{lcccc}
\hline \multicolumn{1}{c}{ Assay } & Primer & Sequence & $\begin{array}{c}\text { Annealing } \\
\text { temperature }\left({ }^{\circ} \mathrm{C}\right)\end{array}$ & $\begin{array}{c}\text { Final primer } \\
\text { concentration }(\mu \mathrm{M})\end{array}$ \\
\hline An. stephensi & KdrF & GGACCAYGATTGCCAAGAT & 50 & 1.25 \\
& VGS_1R & CGAAATTGGACAAAAGCAAGG & 50 & 1.25 \\
An. arabiensis & Agd1 & ATAGATTCCCCGACCATG & 52 & 1.25 \\
& Agd2 & AGACAAGGATGATGAACC & 52 & 1.25 \\
Culex & Cpp1 & CCTGCCACGGTGGAACTTC & 58 & 1 \\
& Cpp2 & GGACAAAAGCAAGGCTAAGAA & 58 & 1 \\
\hline
\end{tabular}




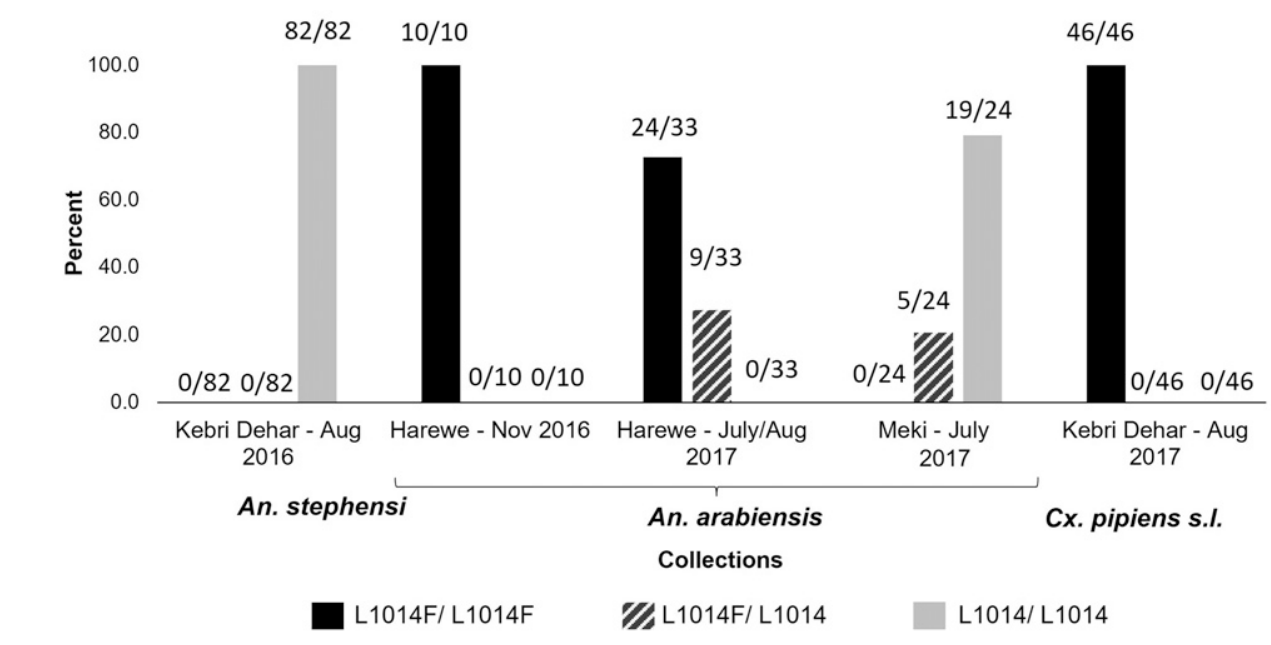

FIGURE 2. Frequency of kdr 1014 genotypes in An. stephensi, Culex pipiens s.l., and An. arabiensis collections.

\section{DISCUSSION}

Our results revealed variations at the $k d r$ locus across different vector species found in eastern Ethiopia that suggest that the role of the target-site mechanism in pyrethroid/DDT resistance varies across species. Notably, the kdr L1014F mutation was not observed consistently across the species included in this study. Unlike the An. stephensi, which carried no L1014F mutations, both $C x$. pipiens s.l. and $A n$. arabiensis carried L1014F. Based on these findings, it is likely that $C x$. pipiens s.l. and An. arabiensis do not share the same resistance mechanism profile as An. stephensi (i.e., $k d r$ target-site resistance is not relevant in all species from a single region). We also observed differences in the nucleotide diversity of the neighboring intronic region of the three species. Although An. stephensi exhibited multiple segregating sites and resultant haplotypes, only a single intronic haplotype is observed for An. arabiensis and $C x$. pipiens s.l. These data may point to distinct differences in biological and environmental factors that shape each species/population. From a species standpoint, behaviors shaped by both their biology and environment, like feeding and resting preferences, may impact the degree of exposure

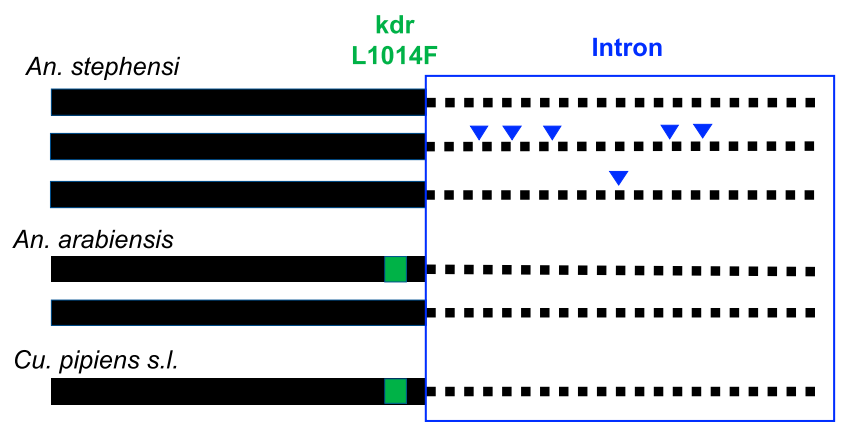

FIGURE 3. Summary of $k d r$ haplotypes across three Culicidae species in eastern Ethiopia. Solid lines depict the exon housing of the $k d r$ locus and dotted lines depict the downstream intron. Green square indicates the presence of $k d r$ L1014F. Triangles denote single nucleotide polymorphisms (SNPs) found in the intron relative to the most prevalent intron haplotype. This figure appears in color at www. ajtmh.org. to insecticides. ${ }^{32}$ Given the variations in resting behavior among the mosquito species represented here (e.g., An. arabiensis is more exophilic ${ }^{33,34}$ and $A n$. stephensi is more endophilic ${ }^{35}$ ), variations in exposure to insecticides may occur and may impact the emergence of $k d r$ mutations. During our study, we expected that endophilic mosquitoes would have greater exposure to insecticides; therefore, An. stephensi would have stronger selective pressure on the $k d r$ locus, leading to less variation. However, the opposite was observed during our study, which causes us to question the feeding and biting behaviors of eastern Ethiopian An. stephensi. ${ }^{32}$ It is unclear if the level of insecticide used in the areas surveyed during this study was enough to impact the biting behavior of $A n$. stephensi. More information about eastern Ethiopian An. stephensi biting and resting behaviors can elucidate behavioral adaptations to insecticides in An. stephensi.

In addition to species-level differences, the different patterns of $k d r$ variations may be explained by multiple evolutionary processes acting on each population sampled. The data may reflect different levels of selective pressure occurring at each location, such that the populations that were undergoing selective pressure from insecticides associated with malaria control or agricultural activities exhibited $k d r$ mutations and no intronic variation. We know that DDTbased and pyrethroid-based insecticides and pesticides have a long history of use in the country, ${ }^{15,36,37}$ although the data about their use at the local level in eastern Ethiopia are quite limited. The variations may also reflect previous demographic events, like recent decreases in population size or population introductions resulting in a bottleneck and a

TABLE 2

Genetic diversity estimates for $k d r$ neighboring downstream introns in the vgsc for An. stephensi, An. arabiensis, and Cx. pipiens s.l.

\begin{tabular}{lrlllll}
\hline Species & $\mathrm{n}$ & $\mathrm{S}$ & $\mathrm{k}$ & $\mathrm{Pi}$ & $\mathrm{h}$ & $\mathrm{Hd}$ \\
\hline An. stephensi & 262 & 6 & 0.996 & 0.00545 & 3 & 0.225 \\
An. arabiensis & 134 & 0 & 0 & 0 & 1 & 0 \\
$\mathrm{Cx}$. pipiens s.I. & 84 & 0 & 0 & 0 & 1 & 0 \\
\hline $\mathrm{n}=$ number of genes (two per individual); $\mathrm{S}=$ number of polymorphic (i.e., segregating) \\
sites; K = average number of pairwise nucleotide differences; $\mathrm{Pi}=$ nucleotide diversity; \\
$\mathrm{h}=$ number of haplotypes; $\mathrm{Hd}=$ haplotype diversity.
\end{tabular}


TABLE 3

Neutrality tests for downstream $k d r$ introns for An. stephensi

\begin{tabular}{lc}
\hline \multicolumn{1}{c}{ Test } & Estimate \\
\hline $\mathrm{n}$ & 258 \\
Tajima's D & 0.03839 \\
Fu's F & 3.556 \\
Fu and Li's D & 1.04354 \\
Fu and Li's F & 0.82943 \\
\hline All $P>0.10$. &
\end{tabular}

decrease in intronic variation. We can best evaluate these possibilities in the context of variations in other regions of the genomes in these mosquitoes. Cytochrome oxidase subunit I (COI) has been previously analyzed in An. arabiensis and $A n$. stephensi. ${ }^{3,22}$ Although multiple COI haplotypes were observed for each $A n$. arabiensis collection, ${ }^{22}$ only a single $\mathrm{CO}$ haplotype was identified in $A n$. stephensi. ${ }^{3}$ The higher level of diversity in $\mathrm{COI}$ in $\mathrm{An}$. arabiensis relative to the $k d r$ intronic region supports that selective pressure rather than a population bottleneck has shaped the variation at the $k d r$. The opposite pattern observed in An. stephensi provides greater support for the absence of selection on that locus in that species. The degree of variation at $k d r$ in An. stephensi may also reflect the likely notion of this species being a recent introduction to that region; ${ }^{38}$ therefore, it would not have had the same number of years of exposure to the local pressure that would cause evolved target-site resistance in the local vector populations. No COI data were available for the $C x$. pipiens s.l. in this study, and both population bottleneck and/or selection on the $k d r$ locus remain plausible explanations for the lack of variation. The multiple collections that comprised our An. arabiensis sample set provide preliminary insight into the basis for population $k d r$ variations within a species. We observed a range of $k d r$ allele frequencies across the An. arabiensis sample collections. The collections differ by location and/or date of collection, suggesting that geography or timing could have a role in the variations in $k d r$ L1014F frequencies observed. Insecticide resistance phenotypes and mutations have been shown to vary within a single population, ${ }^{39-41}$ which may correspond to the seasonal use of insecticides for vector control or agricultural activities. Additional surveillance in a larger sample size is needed to verify the importance of geographic and temporal factors shaping the frequency of the mutation. Another notable observation was the shared intron haplotype between the An. arabiensis that carried the L1014F mutation to those that did not. The mosquitoes that carried the once advantageous allele may experience fitness costs in the absence of the selective pressure, which would result in a rebound of the wild-type allele at that locus. These findings highlight the value of investigating the $k d r$ intronic variation for evidence of fluctuating selective pressures and the potential for the emergence of insecticide resistance in the future.

Our findings have important implications for the molecular surveillance of target-site pyrethroid resistance mechanisms. An analysis of the $k d r$ intronic variation provides important evolutionary historical context to the observed high or low frequency of the $k d r$ mutations that have programmatic implications. In this study, whether $k d r$ mutations were observed at a high frequency (e.g., Harewe in 2016 and 2017) or low frequency (e.g., Meki in July 2017), low intronic variation may be a signal of past selective pressure and the potential for target-site resistance to emerge rapidly. In the future, molecular surveillance should include an analysis of $k d r$ intronic variation to better predict the responses to insecticides by the various vector species present. In addition, comparing $k d r$ frequencies across species sheds light on how the introduction of a new vector species population could lead to differing resistance mechanism profiles across the species targeted for integrated vector control. In this case, the absence of $k d r$ in the An. stephensi population (likely influenced by the variable exposure to insecticides on its path to introduction into eastern Ethiopia) suggests that standing resistance because of metabolic mechanisms would not be compounded by the presence of target-site resistance in An. stephensi, the opposite scenario whereby a new vector introduces resistant mutations could be possible in other settings. Therefore, vector control strategies must account for the unique and dynamic population insecticide exposure histories that invasive vectors have compared with native species in the context of local interventions targeting multiple species. An analysis of $k d r$ genetic variations will provide critical information that will help achieve this goal.

Several limitations to these studies should be considered. The An. stephensi was collected as larvae and pupae and the $A n$. arabiensis and $C x$. pipiens s.l. were collected as wildcaught adults. This method of collection may pose a concern that the immature specimen set would not reflect the natural diversity of the wild-caught adult population. Concerns with clonality, however, are lowered when considering the level of diversity observed at the An. stephensi kdr locus and at the ace-1R locus (three haplotypes detected; data not shown). In addition, although $A n$. stephensi phenotypic resistance has been reported for eastern Ethiopia, phenotypic data regarding $A n$. arabiensis and its association with $k d r$ has only been studied for portions of the country outside of eastern Ethiopia. Furthermore, the association of $k d r$ mutations and phenotypic resistance in Cx. pipiens s.l. observed in other parts of the world have not been confirmed in Ethiopia. Follow-up studies would benefit from additional bioassay tests for An. arabiensis and $C x$. pipiens in eastern Ethiopia in conjunction with the molecular analysis of $k d r$. Finally, because of the geographic variation in $k d r$ mutation frequencies observed in An. arabiensis, future studies should examine the frequency of $k d r$ mutations of these vectors in other regions in Ethiopia to confirm the status of target-site pyrethroid/DDT resistance.

In conclusion, the different patterns of diversity at the $k d r$ loci across species support the notion that Culicidae in eastern Ethiopia likely have different profiles of pyrethroid/DDT resistance mechanisms. Both $A n$. arabiensis and $C x$. pipiens sample sets revealed notable L1014F allele frequencies that confer target-site resistance and the absence of intron variations that may be caused by positive selective pressure on that locus in those species. Additional investigations are needed to confirm other resistance mechanisms (metabolic, cuticle, or another undiscovered mechanism) and the genetic basis of pyrethroid resistance in An. stephensi. Coordinated studies of agricultural and vector control practices with molecular surveillance would also enhance our ability to evaluate the cause of $k d r$ variation observed here. These findings emphasize the need for careful consideration of molecular approaches used to evaluate the insecticide resistance status across multiple species and will inform the 
development and future implementation of novel integrated vector control strategies.

Received October 15, 2020. Accepted for publication October 8, 2021.

Published online January 10, 2022.

Note: The datasets supporting the conclusions of this article are included within the article and its supplemental files. Sequences have been included with this manuscript as Supplemental File 1. Supplemental file appears at www.ajtmh.org.

Acknowledgments: We thank Mr. Negib Abdi and Habtamu Atlaw for their financial support and arranging for the car for the field work. We thank Mr. Geleta Bekele for his technical support rearing mosquitos at the field laboratory. We thank Dr. Jason Pitts and Ms. Jeanne Samake for the helpful comments regarding the manuscript. We acknowledge the support of various entities of the University of North Carolina at Charlotte, including the Department of Bioinformatics and Genomics of the College of Computing and Informatics and the Department of Biological Sciences of the College of Literature Science and the Arts. We are grateful for the support of the Belk Family. We acknowledge the support of Baylor University Department of Biology of the College of Arts and Sciences.

Financial support: This study was financially supported by Jigjiga University. This project was also funded by Baylor University and the University of North Carolina at Charlotte Multicultural Postdoctoral Fellowship.

Authors' addresses: Tarmar E. Carter, Baylor University College of Arts and Sciences, Biology, Waco, TX, E-mail: tamar_carter@baylor. edu. Araya Gebresilassie, Department of Zoological Sciences, Addis Ababa University, Addis Ababa, Ethiopia, E-mail: arayagh2006@ yahoo.com. Shantoy Hansel, Callum Montgomery, Karen Lopez, and Daniel Janies, University of North Carolina at Charlotte, Department of Bioinformatics and Genomics, Charlotte, NC, E-mails: shansel@ uncc.edu, cmontg24@uncc.edu, klopez1@uncc.edu, and djanies@ uncc.edu. Lambodhar Damodaran, University of Georgia, Institute of Bioinformatics, Athens, GA, E-mail: lambodhar.damodaran@uga. edu. Victoria Bonnell, Pennsylvania State University Department of Biochemistry and Molecular Biology, Department of Molecular Biology and Biochemistry, Hershey, PA, E-mail: vab18@psu.edu. Solomon Yared, Jigjiga University, Department of Biology, Jigjiga, Somali Region, Ethiopia, E-mail: solyar2005@yahoo.com.

This is an open-access article distributed under the terms of the Creative Commons Attribution (CC-BY) License, which permits unrestricted use, distribution, and reproduction in any medium, provided the original author and source are credited.

\section{REFERENCES}

1. World Health Organization, 2020. World Malaria Report 2020. Geneva, Switzerland: WHO.

2. Balkew M et al., 2020. Geographical distribution of Anopheles stephensi in eastern Ethiopia. Parasit Vectors 13: 35.

3. Carter TE, Yared S, Gebresilassie A, Bonnell V, Damodaran L, Lopez K, Ibrahim M, Mohammed S, Janies D, 2018. First detection of Anopheles stephensi Liston, 1901 (Diptera: culicidae) in Ethiopia using molecular and morphological approaches. Acta Trop 188: 180-186.

4. Faulde MK, Rueda LM, Khaireh BA, 2014. First record of the Asian malaria vector Anopheles stephensi and its possible role in the resurgence of malaria in Djibouti, Horn of Africa. Acta Trop 139: 39-43.

5. Seyfarth M, Khaireh BA, Abdi AA, Bouh SM, Faulde MK, 2019. Five years following first detection of Anopheles stephensi (Diptera: Culicidae) in Djibouti, Horn of Africa: populations established-malaria emerging. Parasitol Res 118: 725-732.

6. Golding N, Wilson AL, Moyes CL, Cano J, Pigott DM, Velayudhan R, Brooker SJ, Smith DL, Hay SI, Lindsay SW, 2015. Integrating vector control across diseases. BMC Med 13: 249.

7. Ranson H, Lissenden $\mathrm{N}, 2016$. Insecticide resistance in African Anopheles mosquitoes: a worsening situation that needs urgent action to maintain malaria control. Trends Parasitol 32: 187-196.

8. Ranson H, N'Guessan R, Lines J, Moiroux N, Nkuni Z, Corbel $\mathrm{V}, 2011$. Pyrethroid resistance in African anopheline mosquitoes: what are the implications for malaria control? Trends Parasitol 27: 91-98.

9. Davies TG, Field LM, Usherwood PN, Williamson MS, 2007. A comparative study of voltage-gated sodium channels in the insecta: implications for pyrethroid resistance in Anopheline and other Neopteran species. Insect Mol Biol 16: 361-375.

10. Scott JG, Yoshimizu MH, Kasai S, 2015. Pyrethroid resistance in Culex pipiens mosquitoes. Pestic Biochem Physiol 120: 68-76.

11. Ranson $\mathrm{H}$, Jensen $B$, Vulule JM, Wang $X$, Hemingway J, Collins $\mathrm{FH}, 2000$. Identification of a point mutation in the voltagegated sodium channel gene of Kenyan Anopheles gambiae associated with resistance to DDT and pyrethroids. Insect Mol Biol 9: 491-497.

12. David JP, Ismail HM, Chandor-Proust A, Paine MJ, 2013. Role of cytochrome P450s in insecticide resistance: impact on the control of mosquito-borne diseases and use of insecticides on Earth. Philos Trans R Soc Lond B Biol Sci 368: 20120429.

13. Alemayehu $E$ et al., 2017. Mapping insecticide resistance and characterization of resistance mechanisms in Anopheles arabiensis (Diptera: Culicidae) in Ethiopia. Parasit Vectors 10: 407.

14. Asale A, Getachew Y, Hailesilassie W, Speybroeck N, Duchateau L, Yewhalaw D, 2014. Evaluation of the efficacy of DDT indoor residual spraying and long-lasting insecticidal nets against insecticide resistant populations of Anopheles arabiensis Patton (Diptera: Culicidae) from Ethiopia using experimental huts. Parasit Vectors 7: 131.

15. Balkew M, Ibrahim M, Koekemoer LL, Brooke BD, Engers H, Aseffa A, Gebre-Michael T, Elhassen I, 2010. Insecticide resistance in Anopheles arabiensis (Diptera: Culicidae) from villages in central, northern and south west Ethiopia and detection of kdr mutation. Parasit Vectors 3: 40.

16. Yewhalaw D, Van Bortel W, Denis L, Coosemans M, Duchateau L, Speybroeck N, 2010. First evidence of high knockdown resistance frequency in Anopheles arabiensis (Diptera: Culicidae) from Ethiopia. Am J Trop Med Hyg 83: 122-125.

17. Enayati A, Hanafi-Bojd AA, Sedaghat MM, Zaim M, Hemingway $\mathrm{J}, 2020$. Evolution of insecticide resistance and its mechanisms in Anopheles stephensi in the WHO Eastern Mediterranean Region. Malar J 19: 258.

18. Yared S, Gebressielasie A, Damodaran L, Bonnell V, Lopez K, Janies D, Carter T, 2020. Insecticide resistance in Anopheles stephensi in Somali region, eastern Ethiopia.

19. Tajima F, 1989. Statistical method for testing the neutral mutation hypothesis by DNA polymorphism. Genetics 123: 585-595.

20. Chang $X$ et al., 2016. Landscape genetic structure and evolutionary genetics of insecticide resistance gene mutations in Anopheles sinensis. Parasit Vectors 9: 228.

21. Weill M, Chandre F, Brengues C, Manguin S, Akogbeto M, Pasteur N, Guillet P, Raymond M, 2000. The kdr mutation occurs in the Mopti form of Anopheles gambiae s.s. through introgression. Insect Mol Biol 9: 451-455.

22. Carter TE, Yared S, Hansel S, Lopez K, Janies D, 2019. Sequence-based identification of Anopheles species in eastern Ethiopia. Malar J 18: 135.

23. Madden T, 2002. The BLAST Sequence Analysis Tool. Bethesda, MD: National Institutes of Health.

24. Singh OP, Dykes CL, Lather M, Agrawal OP, Adak T, 2011. Knockdown resistance (kdr)-like mutations in the voltagegated sodium channel of a malaria vector Anopheles stephensi and PCR assays for their detection. Malar $J$ 10: 59.

25. Verhaeghen K, Van Bortel W, Roelants P, Backeljau T, Coosemans $\mathrm{M}, 2006$. Detection of the East and West African kdr mutation in Anopheles gambiae and Anopheles arabiensis from Uganda using a new assay based on FRET/Melt Curve analysis. Malar J 5: 16.

26. Chen $L$ et al., 2010. Molecular ecology of pyrethroid knockdown resistance in Culex pipiens pallens mosquitoes. PLoS One 5: e11681. 
27. Rozas J, Sanchez-DelBarrio JC, Messeguer X, Rozas R, 2003. DnaSP, DNA polymorphism analyses by the coalescent and other methods. Bioinformatics 19: 2496-2497.

28. Stephens M, Smith NJ, Donnelly P, 2001. A new statistical method for haplotype reconstruction from population data. Am J Hum Genet 68: 978-989.

29. Scheet $P$, Stephens $M, 2006$. A fast and flexible statistical model for large-scale population genotype data: applications to inferring missing genotypes and haplotypic phase. $A m \mathrm{~J}$ Hum Genet 78: 629-644.

30. Fu YX, 1997. Statistical tests of neutrality of mutations against population growth, hitchhiking and background selection. Genetics 147: 915-925.

31. Fu YX, Li WH, 1993. Statistical tests of neutrality of mutations. Genetics 133: 693-709.

32. Gatton ML et al., 2013. The importance of mosquito behavioural adaptations to malaria control in Africa. Evolution 67: 1218-1230.

33. Tirados I, Costantini C, Gibson G, Torr SJ, 2006. Blood-feeding behaviour of the malarial mosquito Anopheles arabiensis: implications for vector control. Med Vet Entomol 20: 425-437.

34. Kibret S, Wilson GG, 2016. Increased outdoor biting tendency of Anopheles arabiensis and its challenge for malaria control in central Ethiopia. Public Health 141: 143-145.

35. Reisen W, 1986. Population dynamics of some Pakistan mosquitoes: the impact of residual organophosphate insecticide spray on anopheline relative abundance. Ann Trop Med Parasitol 80: 69-75.
36. Mekonen S, Ambelu A, Negassa B, Spanoghe P, 2017. Exposure to DDT and its metabolites from khat (Catha edulis) chewing: consumers risk assessment from southwestern Ethiopia. Regul Toxicol Pharmacol 87: 64-70.

37. WHO, 2007. Implementation of indoor residual spraying of insecticides for malaria control in the World Health Organization, African Region.

38. Carter TE, Yared S, Getachew D, Spear J, Choi SH, Samake JN, Mumba P, Dengela D, Yohannes G, Chibsa S, 2021. Tracking of Anopheles stephensi in Ethiopia using mitochondrial DNA reveals pattern of spread. bioRxiv. doi: https://doi. org/10.1101/2021.04.07.437873.

39. Diabate A, Baldet T, Chandre F, Akoobeto M, Guiguemde TR, Darriet F, Brengues C, Guillet P, Hemingway J, Small GJ, 2002. The role of agricultural use of insecticides in resistance to pyrethroids in Anopheles gambiae s.l. in Burkina Faso. Am $J$ Trop Med Hyg 67: 617-622.

40. Taskin BG, Dogaroglu T, Kilic S, Dogac E, Taskin V, 2016. Seasonal dynamics of insecticide resistance, multiple resistance, and morphometric variation in field populations of Culex pipiens. Pestic Biochem Physiol 129: 14-27.

41. Awolola T, Oduola A, Oyewole I, Obansa J, Amajoh C, Koekemoer L, Coetzee M, 2007. Dynamics of knockdown pyrethroid insecticide resistance alleles in a field population of Anopheles gambiae s.s. in southwestern Nigeria. $J$ Vector Borne Dis 44: 181. 